Cinémas

Revue d'études cinématographiques

Journal of Film Studies

\title{
Cinéma, cinématisme et ciné-littérature en Russie
}

\section{Leonid Heller}

Volume 11, numéro 2-3, printemps 2001

Eisenstein dans le texte

URI : https://id.erudit.org/iderudit/024852ar

DOI : https://doi.org/10.7202/024852ar

Aller au sommaire du numéro

\section{Éditeur(s)}

Cinémas

ISSN

1181-6945 (imprimé)

1705-6500 (numérique)

Découvrir la revue

Citer cet article

Heller, L. (2001). Cinéma, cinématisme et ciné-littérature en Russie. Cinémas, 11(2-3), 167-196. https://doi.org/10.7202/024852ar

\section{Résumé de l'article}

On a coutume d'envisager les rapports entre cinéma et littérature en postulant l'influence du premier sur la seconde. Le présent article propose un autre point de vue. Il rappelle le débat autour de la "réorganisation du système des arts ", qui a fait suite à l'apparition du cinéma. Il montre ensuite qu'une nouvelle sensibilité visuelle naît entre les deux siècles, notamment dans la littérature, avec ou sans un lien univoque avec le cinéma. Eisenstein a appelé " cinématisme " l'ensemble des procédés visuels élaborés par la littérature "précinématographique " qu'il a analysés dans une série d'oeuvres de Pouchkine, Gogol, Zola. Contrairement à Chklovski, qui insiste sur les différences de matériaux entre les deux " médias ", Eisenstein distingue dans l'évolution du cinéma des périodes qu'il rattache à l'évolution littéraire. Ces débats sont ici discutés et confrontés à une autre conception du cinématisme, mise en pratique par des auteurs modernistes comme Boulgakov ou Zamiatine.
Ce document est protégé par la loi sur le droit d'auteur. L'utilisation des services d’Érudit (y compris la reproduction) est assujettie à sa politique d'utilisation que vous pouvez consulter en ligne.

https://apropos.erudit.org/fr/usagers/politique-dutilisation/ 


\section{Cinéma, cinématisme et ciné-littérature en Russie ${ }^{1}$}

\section{Leonid Heller}

\section{RÉSUMÉ}

On a coutume d'envisager les rapports entre cinéma et littérature en postulant l'influence du premier sur la seconde. Le présent article propose un autre point de vue. Il rappelle le débat autour de la "réorganisation du système des arts", qui a fait suite à l'apparition du cinéma. Il montre ensuite qu'une nouvelle sensibilité visuelle naît entre les deux siècles, notamment dans la littérature, avec ou sans un lien univoque avec le cinéma. Eisenstein a appelé "cinématisme" l'ensemble des procédés visuels élaborés par la littérature "précinématographique" qu'il a analysés dans une série d'œuvres de Pouchkine, Gogol, Zola. Contrairement à Chklovski, qui insiste sur les différences de matériaux entre les deux "médias", Eisenstein distingue dans l'évolution du cinéma des périodes qu'il ratcache à l'évolution littéraire. Ces débats sont ici discutés et confrontés à une autre conception du cinématisme, mise en pratique par des auteurs modernistes comme Boulgakov ou Zamiatine.

\footnotetext{
ABSTRACT

When thinking about the relationship between film and literature, we usually focus on the influence of the former upon the latter. This article proposes a different perspective. It evokes the debate over the "reorganization of the artistic system" which arose after cinema's birth. It then demonstrates that a new visual sensibility arose at the turn of the century, particularly in literature, whether this literature had any direct link to the cinema or not. Eisenstein called the visual techniques
} 
found in "pre-cinematographic" literature "cinematism", and analysed these techniques in a number of works by Pushkin, Gogol, and Zola. Unlike Shklovskii, who insisted on the formal differences berween the two media, Eisenstein located periods within film history which co-related to literary history. These debates are discussed here and contrasted with another conception of cinematism, that developed by modernist authors such as Bulgakov and Zamiatin.

\section{Une nouvelle sensibilité visuelle}

Le cinéma et la littérature dans les années vingt, en Russie, ou plus exactement, la rétroaction qui unit les deux domaines artistiques, tel est le sujet de cet article.

En 1926, Boris Eikhenbaum, un des chefs de file de l'école formaliste, écrivait :

Lartirance du cinéma pour la littérature doit être considérée non seulement comme le résultat de tendances syncrétiques, mais également comme une lutte de pouvoir $[\ldots]$. Face au cinéma, la littérature doit prendre à nouveau conscience de ses moyens. Le développement du cinéma repose le vieux problème du rapport et de la différenciation entre les arts ("Littérature et cinéma", dans Albera, 1996, p. 206-207).

Et de remarquer que «la culture cinématographique, comme marque d'une époque, s'oppose à la culture du mot, à la culture livresque et théâtrale qui dominait au siècle dernier». Ces quelques phrases résument les enjeux des rapports entre la littérature et le cinéma tels qu'ils sont vus et débattus dans les années vingt, en Russie. En fait, leur portée est plus générale - on se permettra quelques remarques à ce propos, avant de revenir à notre sujet.

L'arrivée du cinéma vient de bouleverser le système complexe et hiérarchisé des arts (comme l'ont fait avant, et à des degrés différents, la photographie, la révolution picturale déclenchée par les impressionnistes, la découverte du non-objectif dans la poésie). Du coup, la redistribution des places donne le signal à une compétition qui entraîne les arts à creuser et à redéfinir leurs spécificités; mais il leur faut aussi activer leurs composan- 
tes syncrétiques, car chaque art englobe de tels éléments «allogènes" - le gestuel, le visuel, le plastique dans l'écriture, le narratif, le verbal dans le cinéma, etc. - , grâce à quoi un échange est possible à l'intérieur du système.

Le mécanisme est agonique: il déstabilise la hiérarchie des arts et déclenche un combat. Du point de vue formaliste il constitue, à côté du cycle de désautomatisation des formes, le grand moteur de l'évolution artistique. Pour d'autres, cette dynamique aura des implications différentes. Depuis les débuts du modernisme, on parle beaucoup de la «synthèse des arts». On imagine un "art synthétique» qui bénéficie de divers apports interdisciplinaires (la prose symphonique d'André Biély, le théâtre de Meyerhold). On voit la synthèse comme une collaboration équilibrée entre les domaines voisins; ainsi le groupe de Jivskoulptarkh - peinture-sculpture-architecture - conçoit en 1919 1920 un projet parallèle à celui de Bruno Taut et de ses amis ${ }^{2}$. D'autres enfin continuent à explorer l'unité profonde des arts en espérant aboutir à l'« œuvre d'art totale», Gesamtkunstwerk; ils rêvent parfois, à l'instar des symbolistes - Skriabine, Viatcheslav Ivanov, Florenski - mieux que d'une synthèse wagnérienne, d'un acte créateur mystique qui dépassera les limites de l'activité artistique: la doctrine du peintre Vassili Tchekryguine sur la "synthèse vivante de tous les arts dans l'homme" offre une bonne illustration de cette démarche transplantée dans l'époque postrévolutionnaire ${ }^{3}$.

Toutes ces recherches se croisent parfois et se confondent. Le cinéma stimule beaucoup d'entre elles, mais son influence est surtout sensible dans le domaine de la littérature. La situation est relativement bien connue ${ }^{4}$. Mais elle n'a pas vraiment été analysée dans une perspective littéraire. En règle générale, l'intérêt pour ces questions se manifeste davantage du côté des spécialistes du cinéma. En poursuivant leurs objectifs, ils préferent examiner les écrivains directement engagés dans la production de films et les textes pour ou sur le cinéma.

D'autre part, lorsqu'un littéraire met en parallèle la poétique du vers et celle du cinéma, il se sent obligé de rechercher les preuves documentaires de la passion que ce dernier devait inspirer au poète ${ }^{5}$. Cette approche présuppose l'influence directe du 
cinéma sur les gens des lettres; sans doute légitime, elle peut conduire à la restriction du champ d'exploration. Nous proposons au contraire de l'élargir et nous abandonnons le postulat de l'impact initial du cinéma pour expliquer l'apparition des formes d'écriture "cinématographique" (notre regard, répétons-le, est celui d'un historien de la littérature).

Certes, il faut d'abord repasser par les pistes déjà frayées. Dans un article important au sujet des "écrivains russes et le cinéma", Jerry Heil (1986) évoque Gorki, Andréiev, Maïakovski, Meyerhold, Blok, Biély, Brik, Chklovski, Olécha, Tynianov, Babel. La liste est longue et cependant lacunaire. À côté d'innombrables artisans de deuxième plan, tel Lev Nikouline, prosateur et scénariste prolifique, elle rélègue dans l'oubli les figures plus importantes de Marienhof et de Cherchénévitch, chefs de file de l'imaginisme et auteurs de scénarios célèbres ${ }^{6}$, sans parler des écrivains aussi décisifs que Zamiatine, Boulgakov, Erdman, Platonov.

Mais peut-on être exhaustif? En effet, si les écrivains de quelque renom qui n'ont pas été attirés, d'une façon ou d'une autre, vers le travail au cinéma sont rares, plus rares encore sont ceux qui ont choisi de le bouder. Sa présence se reflète dans la poésie, depuis l'affecté mais très évocateur Un midi de juillet (Iiul'skij polden', 1911) d'Igor Sévérianine et le doucement ironique Cinématographe (Kinematograf, 1912) d'Ossip Mandelstam, jusqu'à Mikhail Kouzmine qui accueille, dans La Truite rompt la glace (Forel' razbivaet led, 1927) les ombres de Nosferatu et de Caligari. Si la poésie moderniste engage un dialogue avec le cinéma, c'est encore plus vrai pour la prose. Sans doute serait-il utile et intéressant de compléter la liste de Jerry Heil. Mais il est tout aussi impératif de problématiser les critères et les buts de la recherche.

Rappelons que la réflexion sur le cinéma date en Russie de bien avant la révolution. Si l'on en croit certains historiens, il aurait même attiré l'attention des grands artistes et provoqué un débat sur son statut artistique plus tôt qu'en Europe ${ }^{7}$. Difficile à trancher: des questions analogues sont abordées alors, par exemple, en Allemagne ${ }^{8}$. Il est vrai cependant qu'en Russie le cinéma trouve assez rapidement des commentateurs de grande lucidité et de non moins grande autorité, le critique Korneï Tchou- 
kovski, le poète André Biély, le prosateur Léonid Andréiev. Ils pressentent tous un grand avenir pour ce qu'ils voient comme un mode de communication et de connaissance urbain, démocratique, hauternent technique, et donc moderne, favorisant la confection de nouveaux mythes et l'élaboration de nouvelles formes d'expression.

Après la Révolution, le dialogue littérature-cinéma reprend sur d'autres bases; il ne suffit plus à l'écrivain de noter ses impressions de spectateur ou de faire un pastiche verbal de l'expérience filmique. La vitalité, l'énergie, le potentiel technique du cinéma promettent d'offrir à la littérature, prise d'une fièvre innovatrice, un modèle esthétique régénérateur et l'invitent en même temps sur son propre terrain, un fascinant terrain d'expérimentation. Invitation tentante et dangereuse. Dans l'article cité, Eikhenbaum compare les liens entre la littérature et le cinéma à un mariage où ce dernier joue le rôle du mari arrogant. Et d'ajouter dans un autre texte écrit la même année 1926, au moment où l'on a déjà pu voir Le Cuirassé Potemkine, La Mère, Aélita, Les Aventures de Mister West: «La littérature, vraisemblablement, a besoin d'élaborer des formes libérées de la pensée cinématographique" (Eikhenbaum, "La littérature du point de vue du cinéma", dans Albera, 1996, p. 214). La "contamination" de la littérature par le cinéma doit avoir atteint un degré important pour arriver à une réflexion aussi alarmante et assez énigmatique sortant de la bouche d'un militant formaliste, peu suspect de nostalgie envers l'ancienne "culture du mot".

Une part de responsabilité pour cet état de choses incombe certainement au dialogue que diverses avant-gardes russes nouent avec l'Occident et aux modèles d'importation qu'elles diffusent à cette occasion.

En 1922, le poète et romancier Ilya Ehrenbourg publie Et pourtant elle tourne ( $A$ vse-taki ona vertitsja). Cet hymne à la gloire de la technique moderne - inspiratrice d'un renouveau dans l'art - est aussi le manifeste d'un mouvement qu'Ehrenbourg, lié par un réseau de connaissances personnelles à nombre d'artistes occidentaux, espère lancer à l'échelle mondiale (au même moment, il commence, avec le plasticien suprématisteconstructiviste El Lissitzky, l'édition de Vechtch. Objet. 
Gegenstand, "Revue internationale de l'art moderne», et examine, avec le cinéaste Hans Richter la possibilité de fonder une Internationale constructiviste).

Négligé par les historiens de l'art et de la littérature (trop timoré pour les "vrais" constructivistes, trop osé pour les commentateurs soviétiques), le livre d'Ehrenbourg mérite attention. On y trouve un tableau des traits qui sont censés caractériser la nouvelle culture en train de se construire et un inventaire des groupes et tendances artistiques qui revendiquent leur participation à ce processus: une mise au point synthétique rare à l'époque et, de ce fait, précieuse. Davantage, un chapitre du livre parle du cinéma, et c'est une des premières tentatives postsymbolistes de l'analyser dans une perspective "intermédiale", comme on dirait aujourd'hui. Nous ne pouvons commenter ici ce texte qui a eu, pensons-nous, plus d'influence en son temps qu'il n'est reconnu. Bornons-nous à quelques remarques.

Ehrenbourg ne met pas en doute le statut artistique du nouveau média. Son essence, au-delà de sa nature mécanique, serait la vitesse, et son plus grand créateur, Chaplin, "futuriste" et "constructeur" dont l'art est sorti des cénacles, l'homme le plus populaire de la planète à côté de Lénine. Le rire qu'il suscite est un "grand levier susceptible d'organiser l'humanité". "Les historiens diront: le cinéma fut créé par Edison et Charlie Chaplin» (1922, p. 120 et 128). Ehrenbourg reprend dans son livre les dessins de Charlot que Fernand Léger fit pour La Chaplinade d'Yvan Goll (dans ce "poème cinématographique " de 1920, Charlot est comparé au Christ ${ }^{9}$ ).

Ouvrons une parenthèse: toute l'avant-garde éprouvera cette fascination pour Charlot. La même année en Russie, dans la revue éditée par le constructiviste Aléxeï Gan, Kino-fot, Alexandre Rodtchenko (1922, p. 5-6) note l'apparition de "l'homme nouveau, maître de détails", tels que seront tous les hommes de l'avenir — "aujourd'hui, c'est l'artiste et acteur Charlie Chaplin ". Varvara Stépanova dessine Charlot dans deux numéros consécutifs de Kino-fot, tandis que Viktor Chklovski, chef de file des formalistes, édite en 1923 sa brochure Charlie Chaplin.

Revenons à Ehrenbourg. Selon lui, la leçon de Chaplin, incarnation de l'homme moderne, et de son esthétique - chas- 
sant par son aspect concret les brumes «maeterlinckistes", libérée de la psychologie fumeuse et du moralisme théâtral, dictée par la vitesse, la technicité, la rigueur d'une chorégraphie totalement maîtrisée, la tension permanente - est capitale pour la littérature. Il affirme: "Dans différents pays, devant différents artistes, une nouvelle tâche est apparue, celle de créer le roman cinématographique" (Ehrenbourg, 1922, p. 99, 103 et 104 c'est l'auteur qui souligne).

Il mentionne La Fin du monde (1919) de Blaise Cendrars, également illustré par Léger. Une très rapide comparaison de ce texte avec le poème d'Ivan Goll laisse clairement voir une tendance: interaction image-texte explicitée par la mise en pages; effet stroboscopique obtenu par une narration accélérée et discontinue; féeries de dimensions cosmiques, allégories sociales et politiques confrontées à l'objet et aux scènes de la vie quotidienne; une stylistique (surtout chez Cendrars) de dénomination :

\footnotetext{
Scènes particulières dans différents quartiers. Les artistes à Montparnasse; les élégantes au bois; l'apéro au Moulin-Rouge.

Les Halles à cinq heures du matin. Un encombrement de voitures au Châteler; la Bourse; la sortie des ateliers rue de la Paix [...] (Cendrars, 1919, p. 21-22).
}

L'image littéraire disparaît au profit de l'évocation directe d'actions, de lieux, d'objets; la description est presque totalement abandonnée; le rythme saccadé des phrases souligne le découpage narratif en discontinuité (la formule la plus élémentaire qui s'en laisse déduire - une action/un objet = une phrase $=$ un paragraphe - remonte, nous semble-t-il, aux romans d'aventures d'un Ponson du Terrail ou d'un Louis Boussenard). Dans ses poèmes et reportages des années vingt, Cendrars développera cette stylistique qui sera identifiée comme inspirée du cinéma, tout en travaillant l'aspect documentaliste qui en fera en Russie soviétique une référence obligée pour la recherche factographique (ou, dans le langage formaliste, centrée sur le matériau).

Ce n'est pas un hasard si, plus tard, Eisenstein envisage de tourner un film d'après $L ' O r$ de Cendrars. 
En même temps que Goll et Cendrars, Ehrenbourg évoque Jules Romains et son roman Donogoo Tonka (1920), dont l'ambition annoncée dès son sous-titre fut de fonder un genre prosaïque nouveau, le récit cinématographique (notons entre parenthèses que déjà en 1907, Léonid Andréiev a qualifié en termes identiques une de ses nouvelles satiriques ${ }^{10}$, mais l'exemple n'a pas eu de suite immédiate).

Traduit en russe en 1922, Donogoo Tonka raconte une machination financière qui exploite l' «erreur scientifique" d'un éminent géographe pour provoquer la naissance, quelque part en Amérique du Sud, d'une ville nouvelle abritant un État quasiutopique. C'est un vrai "film d'aventures». L'action se transporte instantanément d'un bout du monde à l'autre. La description se fait uniquement de l'extérieur, elle privilégie le geste et le décor. Lécriture transpose explicitement le langage du cinéma en l'imitant et en le parodiant (au sens classique de charge, exagération sélective des traits typiques, et au sens tynianovien de régénération de procédés automatisés). La narration, tissée autour d'une intrigue finalement assez molle, se présente comme un fidèle compte-rendu de ce qui est censé se passer sur l'écran. La typographie sépare le texte principal des commentaires, documents incorporés (lettres ou illustrations), répliques; de plus, ceux-ci sont encadrés et figurent ainsi les intertitres de film. Cette «synesthésie", reproduite dans l'édition russe, s'insère organiquement dans le contexte des recherches polygraphiques de l'époque.

Kino-fot publie une critique très favorable de Donogoo Tonka: «La littérature a enfin compris le nouveau rythme de la vie que seul le cinéma percevait jusqu'ici ". " Dans LEF (Front gauche de l'art, dirigé par Maïakovski), dont certaines mises en pages imitent Donogoo Tonka, le théoricien productiviste Boris Kouchner réagit aux formules d'Ehrenbourg et de Romains en définissant la nouveauté du cinéma par sa capacité paradoxale d'exprimer la temporalité du récit à travers les relations spatiales de l'image. Il assure qu' "on va au cinéma non point pour voir, mais pour suivre du regard un récit raconté avec maîtrise et tension" (Kouchner [Kusner], 1923, p. 134). Il propose le concept de "visio-récit " (izopovest) et appelle les écrivains à le pratiquer en prenant des leçons auprès du cinéma ${ }^{12}$. 
Parallèlement, en 1923, Viktor Chklovski, chef de file des formalistes, publie à Berlin (comme Ehrenbourg) un livre intitulé La Littérature et le cinématographe (Literatura i kinematograf): nous y reviendrons. Un peu avant, en janvier 1922, la première livraison d'une nouvelle revue russe berlinoise consacrée au cinéma (Chklovski y contribuera) s'ouvre sur un avantpropos de la rédaction où l'on peut lire la remarque suivante:

\begin{abstract}
"Mon trésor, écrivit Goethe à l'une de ses amantes, ne me compromet pas, ne me salue pas sur Unter-denLinden. " C'est ainsi qu'on traite aujourd'hui le cinéma. On le visite, on y travaille, on l'aime, mais on ne le salue pas.
\end{abstract}

Le nom de la nouvelle revue, L'Art du cinéma (Kino iskousstvo), reflète une attitude nouvelle. Les livres d'Ehrenbourg et de Chklovski, les articles de Rodtchenko et de Kouchner sont bien les signes du changement: on se met à saluer le cinéma sans éprouver de honte. Kino-fot et $L E F$ publient les manifestes de Dziga Vertov - l'avant-garde affirme sa présence à laquelle seront liées les pages les plus illustres de l'histoire du cinéma soviétique.

Tout un courant de "prose cinématographique" inonde l'espace littéraire, des récits se multiplient qui font appel à la stylistique "filmique" — on peut aller jusqu'à voir l'influence de celle-ci dans l'usage massif de la "phrase saccadée» (rublenaja fraza) par la prose dite ornementale - et qui transposent plus ou moins explicitement le langage du cinéma en l'imitant et en le parodiant (au sens classique de charge, exagération sélective des traits typiques, et au sens tynianovien de régénération de procédés automatisés). On pourrait dresser une longue liste de telles œuvres, de La Spécification de l'iditole (1923), « roman de production accéléré" du futuriste Sergueï Bobrov, à Mess Mend (1924) de Marietta Chaguinian et à Ypérite (1925) de Chklovski et Vsevolod Ivanov, en passant par les productions des «frères Sérapion ", groupe de jeunes prosateurs en vogue, et, bien entendu, celles d'Ehrenbourg lui-même, qui consacrera au cinéma plusieurs essais ${ }^{13}$ et à qui l'on devra plusieurs récits, notamment Un film gâché (Isporcennyj fil'm, 1923), reprise parodique du 
modèle "roman-scénario " à la Jules Romains. Le courant sort du lit avant-gardiste.

Diablerie (D’javoliada, 1924) de Mikhaïl Boulgakov revendique clairement les procédés de cinéma qui fonctionnent à différents niveaux du récit et évitent ainsi le littéralisme et la monotonie fonctionnelle (nous le disons sans nuance péjorative) de Donogoo Tonka. Citons quelques passages particulièrement évidents :

Il sauta et s'accrocha à la caténaire d'un tramway. La caténaire le ballotta pendant près de cinq minutes et le jeta à terre devant le bâtiment vert de huit étages [...] La fureur saisit Korotkov. Il brandit le candélabre et en frappa la pendule. Celle-ci lui répondit par un grondement et un jet d'aiguilles en or. Caleçonnière bondit hors de la pendule, se transforma en coq blanc [...] Korotkov sauta sur l'asphalte, regarda autour de lui, saisit trois boules, bondit jusqu'au parapet, l'escalada et regarda en bas. Le cœur lui manqua. Il aperçut devant lui les toitures des maisons, qui semblaient aplaties et toutes petites, une place, sur laquelle des tramways avançaient tout doucement, et la foule semblable à des insectes (Boulgakov, 1971, p. 37, 53 et 57).

Si le but visé par le "récit cinématographique" de Jules Romains est de "transcrire" objectivement un film imaginaire en réduisant la forme du roman à celle du scénario, Boulgakov "transcode" et opère un basculement inverse en intégrant à la narration échevelée l'intériorité de son héros; il faudrait peutêtre qualifier la prose de Diablerie d' "hyper-cinématographique". Outre l'imitation compositionnelle sous la forme de montage et de changements de plans au rythme toujours plus haletant, on peut relever la citation d'images (Harold Lloyd accroché à la caténaire d'un tramway); l'utilisation de prises de vue inhabituelles (en plongée); la reprise de clichés narratifs génériques (coursepoursuite); enfin, la description sur-signifiante, propre au cinéma et aux arts scéniques apparentés (cirque, music-hall, cabaret excentrique). On peut inclure dans cette dernière catégorie les détails en gros plan (horloges, statues, barrières), les personnagesrôles (policier, cocher, directeur), la mécanicité du geste et du mouvement allant jusqu'à la rupture du vraisemblable (carica- 
ture, burlesque), l'organisation spatiale mise en évidence (ascenseurs, couloirs, escaliers, portes, cloisons transparentes), la temporalité en tant qu'itinéraire multiple dans l'espace que les héros perçoivent, pour le traverser ensuite, de préférence en courant, en bondissant, en tombant.

Or, un autre type d'écriture est à trouver dans la production de l'époque; il peut être illustré par un exemple emprunté à un récit de Vladimir Lidine (1923, p. 10), auteur des textes qui reflètent les modes du moment :

Le chef de train mettait des draps propres, frais; derrière une petire table, le représentant jouait au bésique avec l'avocat; le train avançait, avançait, se balançait, rugissait ; arrêts engloutis par l'hiver; stations à l'éclairage livide, kiosques avec des journaux en éventail, chapeau melon d'un commis voyageur planant au-dessus des côtelettes Pojarski. Derrière la station, une ville, noire, perdue $[\ldots]$.

Encore un exemple, dû cette fois à Alexandre Grine (1972, p. 66), écrivain qui, contrairement à Lidine, restait (ou semblait rester) à l'écart de la mode:

D'un geste rapide, elle couvrit son visage d'un mouchoir et ce fut tout ce que j'eus le temps de distinguer nettement. Ensuite les arbres se mirent à défiler, séparés par des rais de lumière qui m'évoquaient tantôt la course d'une silhouette féminine, et tantôt me rappelaient que je galopais moi-même à perdre haleine.

On peut percevoir, dans ces derniers fragments, quelque chose de l'enchaînement des plans dans un film, d'un découpage vigoureux se faisant à même de la phrase. Mais les passages de ce type n'apparaissent dans les récits que par intermittence et la référence au cinéma n'y est pas explicite. On pourrait multiplier les exemples; ceux que nous venons de voir montrent déjà, croyons-nous, qu'il peut être utile d'abandonner certains automatismes qui président à l'étude de la relation cinémalittérature, comme passage obligé par l'avant-garde (les prosateurs en titre de $L E F$ sont moins inventifs que les traditionalistes Boulgakov ou Grine dans l'entreprise de "cinéfication") ou 
priorité du "montage" parmi les "techniques filmiques" (l'aspect paradigmatique avec point de vue, structure et texture de l'image, peut revêtir autant sinon plus d'importance que son aspect syntagmatique: enchaînement de plans, montage) et ainsi de suite.

Notre suite d'exemples semble confirmer également le doute que nous avons formulé au début de cet article et qui nous a amené à abandonner l'idée qui soumet toute l'écriture "cinématographique " à l'expérience directe du cinéma. En effet, si un tel présupposé s'applique bien à la démarche d'un Ehrenbourg ou d'un Chklovski, tient-il encore pour les textes comme ceux de Lidine ou de Grine? La mode de l'«écriture-cinéma " n'a-t-elle pas une cause plus profonde? Afin de pousser plus loin notre analyse, tournons-nous brièvement vers le travail d'Evguéni Zamiatine, écrivain significatif dans ce contexte.

En 1923, dans l'article sur "La nouvelle prose russe", Zamiatine (1989, p. 128) donne une bonne note aux dernières productions d'Ehrenbourg qui trahissent «une indubitable parenté avec la prose française moderne (les dadaïstes, Cendrars, Donogoo-Tonka de Jules Romains)", et réserve une mention particulière à l'un de ses récits, construit comme une imitation de scénario: une nouveauté pour la littérature russe. Dans un panorama de la nouvelle prose qu'il écrit l'année suivante, Zamiatine ("Aujourd'hui et l'époque moderne», 1990, p. 134) qualifie ainsi Diablerie:

Le fantastique, qui plonge ses racines dans le quotidien, vif comme un changement de tableaux au cinéma, est l'un de ces rares cadres formels dans lesquels on peut placer notre hier, les années 1919, 1920. Nous appliquerons le terme "cinéma" à certe œuvre d'autant plus que toute la nouvelle est une surface plane à deux dimensions; tout est en surface et il n'y a aucune profondeur de scène, ne serait-ce que d'un centimètre.

En 1922-1923, Zamiatine collabore étroitement avec Kornéi Tchoukovski, l'un des critiques russes les plus écoutés, dont l'essai «Nat Pinkerton », composé en 1906, analyse brillante et parfois prémonitoire du roman policier et du cinéma ${ }^{14}$, a certainement marqué la culture de masse (en particulier, l'idée du 
"Pinkerton rouge») en train de se forger dans les années vingt. Tchoukovski et Zamiatine préparent une série d'éditions de $\mathrm{O}$. Henry ${ }^{15}$, prosateur américain auquel un grand succès sera promis en Russie. Le premier traduit et commente son roman Of Kings and Cabbage, tandis que le second travaille à un recueil de nouvelles. Et c'est dans la préface à ce dernier qu'il expose le plus longuement ses vues sur le cinéma (Zamiatine, 1990, p. 94-95):

C'est une erreur de dire que le cinématographe a été inventé par Edison: Edison et O'Henry l'ont inventé à eux deux. Dans le cinématographe, il y a avant tout du mouvement - du mouvement coûte que coûte. Et le mouvement, la dynamique, c'est avant tout O'Henry; d'où ses côtés positif et négatif. Le lecteur qui se trouve dans le cinématographe d'O'Henry en ressortira rafraîchi par le rire [...] Mais les effets comiques sont parfois outrés, forcés, grossiers. Il faut à tout prix attendrir le public de temps en temps, au cinéma: O'Henry compose pour lui des drames délicieux de quatre pages. Mais il arrive que ces drames soient sentimentaux et sentencieux du point de vue cinématographique. C'est du reste rare chez O'Henry, il ne s'attendrit qu'une seconde, et le voilà qui galope à nouveau, rieur, moqueur, léger; une langue rapide, un esprit rapide, des sentiments rapides - chaque muscle en mouvement, comme chez cet autre héros national américain, Charlie Chaplin. À quoi Charlie Chaplin croit-il, quelle est la philosophie de Charlie Chaplin? Sûrement à rien, sûrement aucune: il n'a pas le temps.

La position zamiatinienne doit beaucoup à la perception prérévolutionnaire, assez contradictoire, du cinéma. Vitesse de la narration, vitesse des gestes, sentimentalisme, humour, certain primitivisme du contenu, telles seraient ses caractéristiques selon Zamiatine. Biély (1911, p. 251) croyait que la naïveté et la pureté du cinérna le plaçaient plus haut que la littérature contemporaine. Pour Goll, Ehrenbourg, Rodtchenko, nous l'avons vu, Charlot représentait l'essence même de l'homme moderne. Moins exalté, Zamiatine est gêné par la "platitude" bidimensionnelle, par l'absence de la philosophie. Son attitude le 
situe quelque part entre un Mandelstam, qui reconnaissait avec un sourire amusé l'efficacité émotionnelle élémentaire du cinéma-«loubok ${ }^{16}$ ", et un Tchoukovski pour qui le cinéma, produit de la ville moderne, est une sorte de nouvelle épopée à l'usage du terrifiant "sauvage urbain, universel et total ${ }^{17}$ ". En même temps, Zamiatine apprécie la liberté compositionnelle, l'intrigue musclée, le choc produit par le changement éclair de tableaux : sur ce dernier point, il se rapproche de Léon Tolstoï, fasciné par la technique cinématographique, et peut-être de Maïakovski. Le héraut du futurisme saluait l'activisme du film qui allait libérer l'acteur du décor et insuffler de la vie dans les objets.

Si la vision zamiatinienne du cinéma oscille entre le modernisme pré- et l'avant-garde postrévolutionnaire, il fait montre d'un discernement étonnant lorsqu'il se penche sur la pratique de l'écriture. Dans les essais sur l'actualité littéraire mentionnés plus haut et qui ont un caractère programmatique, il définit en peu de mots la différence entre les tentatives "cinéficatrices" d'Ehrenbourg et de Boulgakov. Le premier importe dans la littérature russe un procédé (imitation du scénario). Le second invente une poétique. Ce qui intéresse Zamiatine, c'est la sensation de "fantastique" que la succession rapide d'images confere à la prose (il ne semble pas connaître l'expressionnisme allemand, mais il valorise - à l'inverse d'un Gorki - le côté irréel, fantomatique, des images filmées). Le style cinématographique prend donc naturellement sa place dans un dispositif qui vise cette fusion entre le quotidien et le fantastique (sintez fantastiki $i$ byta) autour de laquelle Zamiatine ("À propos du synthétisme", 1990) voudrait voir se développer une nouvelle école littéraire et artistique, le synthétisme.

L'essai sur $\mathrm{O}$. Henry permet à Zamiatine de formuler une autre idée importante. Il affirme que la gloire d'avoir inventé le cinéma doit être partagée entre Edison et $\mathrm{O}$. Henry. Autrement dit, les traits du récit que nous associons à l'influence du cinéma sont présents dans la manière de l'écrivain new-yorkais et sont déterminés par le caractère de la culture américaine dans son ensemble, par sa technicité et sa rapidité. Zamiatine fait plus qu'adapter à ses besoins les mythes modernistes de Technique et 
de Vitesse : il en poursuit à la fois l'élaboration et l'analyse. Au tandem Edison-Charlot responsable, selon Ehrenbourg, de l'invention du cinéma, il répond par sa formule Edison-O. Henry. Ce qui revient à dire que les procédés "filmiques» du récit sont nés avant le cinéma; ce sont eux qui l'ont fait naître. Le présupposé sur la nature secondaire de toute "ciné-écriture» par rapport à l'expérience du cinéma (qui n'a en tout cas pas pu avoir beaucoup influencé $O$. Henry, mort en 1910), ce présupposé ne tient plus. Devançant bien des analyses et des théories, la formule de Zamiatine s'inscrit dans le débat sur les modalités des échanges entre le cinéma et la littérature.

\section{Eisenstein et Chklovski}

Dans ce débat, les voix les plus fortes et les plus engagées nous semblent être celles de Sergueï Eisenstein et de Viktor Chklovski, deux remarquables théoriciens et en même temps praticiens hors pair (l'écart qui peut apparaître à distance entre le poids respectif de deux hommes dans l'histoire culturelle fut peu perceptible pour les contemporains). Chklovski commente à chaud tous les films d'Eisenstein, l'évoque en permanence dans ses écrits et lui consacre entièrement l'un de ses derniers livres dans lequel il reprend quelques-uns de ses anciens arguments critiques ${ }^{18}$. De son côté, Eisenstein ne cite guère Chklovski et, dans ses Mémoires, le qualifie, avec un brin de dédain, de "voyeur littéraire" (Eisenstein, 1997/1, p. 221); il reconnait cependant sa perspicacité, inclut ses textes dans le programme des cours qu'il donne à l'Institut du cinéma (VGIK) et, plus important, utilise les concepts analytiques introduits par l'auteur formaliste (tel que le «freinage» de l'action). Il n'est pas exclu que le style qu'Eisenstein développé dans les années quarante et qui s'épanouit dans ses mémoires - syntaxe allégée, phrases courtes revenant à la ligne, récit tout en associations et en ruptures - , doive quelque chose aux écrits des années vingt de Chklovski. Ces deux acteurs, emblématiques de l'époque qui vit l'explosion avant-gardiste et le retour à l'ordre stalinien, restent très liés. Faute de place et de compétences pour examiner en profondeur leurs positions, notre propos sera grandement simplifié et fera abstraction de l'évolution historique. 
En 1926 Eisenstein annonce pour le cinéma le début d'une "deuxième période littéraire" (Eisenstein, 1974, p. 162). Lors de la première, le cinéma s'était inspiré des simples modèles narratifs; la deuxième doit marquer le passage à la constitution d'un langage symbolique, d'un véritable discours (rec) cinématographique, capable d'exprimer ce qui ne peut l'être en image. Ce travail doit s'inspirer de la littérature. C'est pourquoi l'analyse de celle-ci constitue un aspect important de la formation et du travail du cinéaste.

Eisenstein déclare qu'exception faite d'Isaac Babel et (ce qui surprend) de l'auteur d'ouvrages documentaires Sofia Fédortchenko ${ }^{19}$, il ne voit guère d'auteurs contemporains capables d'exercer sur le cinéma une influence enrichissante (Eisenstein, 1988 , p. 96-98). En revanche, il voue un véritable culte aux classiques (c'est également le cas pour ses goûts en peinture, comme l'a remarqué François Albera ${ }^{20}$ ). Dès les années trente, il conduit au VGIK des séminaires consacrés à Zola qui, selon lui, constitue "pour un cinéaste, la plus grande école sur le plan méthodologique". Les pages de Zola seraient autant de "feuilles de montage"; on y trouverait des modèles de composition tendue et libre, à l'instar de tableaux d'un Degas ou d'un Manet ( Puskin i kino" [1939], Eisenstein, 1964-1971, t. II, p. 309-311). Ce en quoi Zola s'opposerait à Balzac, auprès duquel (comme auprès de Velasquez) on ira chercher l'inspiration pour des portraits psychologiques: une différence que les étudiants auront à expliquer aux examens. La méthode Eisenstein correspond mot pour mot aux préceptes de Lénine: la culture de la nouvelle société doit utiliser ce qu'il y a de meilleur dans le patrimoine mondial. Pendant près de deux décennies, ce grand érudit et travailleur boulimique alternera, juxtaposera et tentera d'unifier ses analyses d'œuvres et de techniques venant de différentes aires artistiques et culturelles; la littérature, bien sûr, y tient une place de choix.

Il tente de démontrer que les grands artistes et écrivains du passé expriment déjà une vision proche, par son dynamisme et sa plasticité, de ce qui peut être convié par le film. L'observation de Zamiatine se retrouve ainsi sous une forme beaucoup plus élaborée. À cet esprit du cinéma avant la lettre et hors de son 
domaine propre, Eisenstein donnera le nom de cinématisme $e^{21}$. Il aime en chercher les manifestations chez Zola, Maupassant ou Dickens - qui préfigure selon lui toute la poétique de Griffith, avec des jeux analogues d'éclairage, d'accélérés et de ralentis, de contrastes entre agrandissements de détails et scènes d'action (Eisenstein, 1976a, p. 366-382). Mais ce sont, bien entendu, les écrivains classiques russes qu'il aborde le plus souvent, et parmi ceux-là, Pouchkine et Gogol. Il veut tourner un film sur les amours du premier, décortique souvent ses poèmes, organise des travaux pratiques pour analyser les ouvres du second et le titre de l'ouvrage auquel il travaille juste avant sa mort réunit les noms de ces deux géants incontestés et mythiques.

La force directe du style et l'art du "montage poétique" pouchkiniens, la puissance lyrique et pathétique de Gogol et sa richesse de métaphores, telles sont les qualités qu'Eisenstein étudie pour les «recycler» en un langage visuel. Prenons l'exemple de son analyse probablement la plus connue et la plus pertinente. Un passage du poème de Pouchkine Poltava montre Pierre le Grand sortir d'une tente et monter à cheval pour se lancer dans la bataille. Eisenstein démonte l'organisation minutieuse de la séquence de quatorze vers: l'empereur n'apparaît pas tout de suite; au début, c'est sa voix que l'on entend, un moment passe avant qu'il ne se dégage du cortège qui l'entoure, l'attention est attirée d'abord sur ses yeux et sa face terrible, après seulement sur sa silhouette, vue comme en contreplongée, surgit, etc. Eisenstein découpe toute la scène en plansimages; chaque expression poétique est dotée d'un équivalent plastique visuel et cette série visuelle se déroule en décalage par rapport à la "ligne musicale" que constitue le découpage en vers: parfait exemple du montage en contrepoint (Eisenstein, 1958, p. 105-110).

Dans un autre cas d'école, qui devait entrer dans le grand livre sur le montage, Eisenstein transcode de la même manière le passage final du Cavalier de bronze, en en faisant sortir des images dont la composition et surtout la succession sont censées produire le même sens et le même effet que le texte; il y trouve, en plus du montage contrapuntique (ou imbriqué), une composition obéissant au principe de la section d'or. D'après ses calculs 
— inspirés par les recherches des musicologues —, les trois composantes du vers pouchkinien, son phrasé (sa musique), son déroulement sémantique et les images qu'il enchaîne reproduisent chacune la proportion divine (le point fort se situant à peu près à 0,6 de la longueur de la série). D'où la beauté du vers et son impact irrésistible sur le lecteur ${ }^{22}$.

Cette approche en profondeur, multidimensionnelle sinon globalisante, contraste avec les objectifs bien plus circonscrits d'un Lev Kouléchov ${ }^{23}$ ou, plus tard, d'un Mikhail Romm ${ }^{24}$ dans leurs analyses de Gogol, Pouchkine ou Tolstoï. Le premier ne semble en tirer qu'une leçon de montage - et Eisenstein le critique pour cela ${ }^{25}$; le second imite son maître déjà disparu, mais se contente de repérer l'explicite en restant surtout attaché à suivre le mouvement du regard tel qu'il est décrit par le texte. Eisenstein (sur le tard) pense que l'art "consiste en ce que chaque fragment de l'œuvre soit un élément particulier d'un tout organique" ("Odolzajtes' " [1932], dans Eisenstein, 1964-1971, t. II, p. 67), que l'organicité intègre tous les niveaux de l'œuvre et que ses principes sont formalisables. Chaque fragment est signifiant, mais les relations qu'ils entretiennent entre eux le sont encore davantage - en cela, on le sait, il est reconnu par Viatcheslav V. Ivanov et Iouri Lotman comme précurseur de la sémiologie. En réalité, il va bien au-delà de l'étude de codes artistiques. Sa recherche vise moins le fonctionnement du code que l'effet qu'il produit; alors qu'elle n'était encore qu'à ses débuts, Chklovski en a formulé ainsi l'enjeu : "[...] réduire la signification du plan cinématographique, le rendre univoque, pour qu'il ne puisse être déchiffré que d'une seule façon" (Chklovski [Sklovskij], "Osibki i izobretenija » [1928], 1965, p. 100). Ce mirage de la communication (affective) totale, Eisenstein ne cessera de le poursuivre; sa "doctrine" differe de la sémiologie artistique (et non médicale) précisément par l'aspect pragmatique déterministe. Il tend à éliminer le bruit nécessaire à la production de l'information artistique. Lorsqu'il aborde la littérature, on peut qualifier sa démarche non seulement de "pansémantique", mais également de "pan-iconique". C'est ce qui la fragilise. L'inépuisable et géniale imagination pousse Eisenstein à concrétiser chaque élément en une transposition visuelle. 
Paradoxalement, il rompt ainsi l' "organicité " (nous préférons dire la spécificiré) du texte poétique, et sa richesse informative (son caractère indécidable, pour adopter le parler à la mode aujourd'hui). On pourrait le montrer sur de multiples exemples; on se contentera d'un seul, qui nous semble particulièrement frappant. Voici un passage de Tarass Boulba de Gogol (s.d., p. 124), traité par Kouléchov et repris par Eisenstein devant son auditoire du VGIK. Il y est question de l'engagement d'Andriï, fils de Tarass, en chevalier polonais, dans la bataille tout homérique contre les Cosaques:

\begin{abstract}
Andriï, lui, cependant, tout à l'ardeur du combat, plein de désir de mériter le précieux gage dont il se parait, fonçait sur les Cosaques comme un jeune lévrier, le plus beau et le plus rapide de la meute. "Vélaut!" crie le vieux chasseur, et le lévrier s'élance comme une flèche, à une telle allure qu'il semble voler par-dessus terre, penché légèrement sur le côté, soulevant la neige de ses pattes, dépassant dix fois le lièvre lui-même dans l'ardeur de sa course.
\end{abstract}

Reprochant à Kouléchov de n'avoir saisi dans cette comparaison que le rythme le plus conventionnel dans le changement de plans, Eisenstein en dégage une richesse impressionnante d'indications concernant la course, les mouvements du cheval, les mottes de terre arrachées par les sabots, l'assise (penchée sur le côté!) du cavalier... Seul problème: il identifie le lévrier au cavalier, écrase le comparé avec le comparant; or Gogol ne le fait jamais, qui préserve un jeu de dissemblance, une "différance», entre le thème et le phore, le comparé et le comparant. Les images du chevalier et de l'animal ne se confondent pas, elles coexistent et se contredisent (le combat se passe en été, la chasse en hiver, etc. : Nabokov a longuement parlé de cet aspect du génie gogolien ${ }^{26}$.

La spécificité du texte disparaît dans le transcodage, curieusement plus linéaire et "littéral». La lecture des textes littéraires (scénarios) écrits par Eisenstein lui-même laisse également une curieuse impression de "trop dit". En fin de compte, on ne peut s'empêcher de penser qu'il instrumentalise la littérature. La reconnaissance de son grand potentiel lui sert à affirmer la grande 
mission "synthétiste" du cinéma (autrement dit: pour asseoir la préséance de son cinéma "total" sur la littérature). Il croit à l'omnipotence du créateur et de sa volonté, à l'omnipotence du cinéma; le discours généré par le choc des images ouvre la porte vers de nouvelles dimensions, imprime la sensualité, la complétude de la forme sensible au processus intellectuel, à la formule abstraite de la science, mais aussi impose une rigueur idéologique à l'arbitraire de la forme (Eisenstein, 1974, p. 196). Parmi tous les arts, seul le cinéma est capable de le faire; un tel cinéma intellectuel représentera l'apport majeur de la révolution au trésor de la culture mondiale, en créant une synthèse de la science, de l'art et du caractère de classe militant ${ }^{27}$.

Ainsi c'est au cinéma de réaliser le Gesamtkunstwerk dont le rêve hante le modernisme depuis Wagner; le cinéma deviendra un langage "orphique" universel. Nous ignorons si l'on a comparé les systèmes d'Eisenstein et de Scriabine — la ressemblance est grande, surtout si on voit le résumé du projet scriabinien dû à Léonid Sabanéiev, auteur de la première monographie du compositeur ainsi que d'un travail sur l'organisation musicale de la parole ${ }^{28}$ (Eisenstein connaissait bien les deux livres; il puise dans le second sa réflexion sur la section d'or). Vers la fin des années quarante, Eisenstein renoue explicitement avec le discours romantico-symboliste en évoquant " [...] un moment dans la vie d'un artiste véritable quand la prison d'existence pitoyable s'éclaire d'un feu sacré de la vocation» («Neravnodusnaja priroda. III : Pafos" [1946-1947], dans Eisenstein, 1964-1971, t. II, p. 152].

On est pris à la gorge en lisant ce cri de désespoir lancé en plein jdanovisme, mais on conçoit facilement qu'un tel retour vers le symbolisme et la métaphysique de l'art - latent depuis les années trente - ne devait pas être du goût de l'ancien combattant antisymboliste Chklovski, de même que le pan-iconisme appliqué à la littérature ne pouvait plaire au formaliste qui avait commencé sa vie dans la science en infirmant l'équation stéréotypée " poésie = image».

Les analyses littéraires d'Eisenstein n'ont pas davantage convaincu Chklovski. Éloge et réfutation, son livre sur Eisenstein pose à nouveau le problème de fond: est-il licite de faire une 
traduction ou un transcodage du langage de la poésie dans celui du cinéma? C'est la suite d'un débat commencé cinquante ans plus tôt. Face à l'utopie de l'œuvre d'art totale et totalement intégrée dont avaient rêvé les symbolistes, Chklovski (1985, p. 111) donnait alors une définition de l'art en tant que mouvement brownien :

\begin{abstract}
Si l'on met dans un récipient contenant de l'eau une poudre extrêmement fine [...], nous noterons bientôt que ces particules se sont ébranlées. Elles subissent des milliards de chocs moléculaires invisibles qu'elles mettent de cette façon en évidence. J'ignore si cela se produit réellement en physique. Je ne suis pas physicien. Mais dans l'art, il en va bien ainsi, l'art n'est pas créé par une volonté isolée, par un génie isolé, l'hommecréateur n'est que le lieu d'intersection géométrique des lignes et des forces qui ont pris naissance en dehors de lui.
\end{abstract}

Définition plus modeste que celle d'Eisenstein (peut-être aussi plus moderne: le mouvement brownien est un exemple de choix exploité par les théories contemporaines du chaos) et en conséquence, une vision moins ambitieuse de l'art. Chklovski insiste sur la spécificité de chaque domaine artistique et sur l'impossibilité de globaliser et d'unifier les démarches. Il ne se prive pas de suggérer la supériorité de la littérature sur le cinéma et prophétise que le cinéma, phénomène crucial pour l'époque actuelle, disparaîtra dans l'avenir. Son raisonnement est audacieux. La pensée humaine, le mouvement dont est capable l'être humain sont des quantités continues; de même, la parole poétique qui particularise le sens général à travers une série de relations différentielles; et puisqu'elle est latérale, oblique (mimoskazannoe slovo), elle préserve l'intériorité de l'objet ne faisant que la suggérer. L'objet filmé s'impose dans son évidence et sa particularité ; reconnu d'abord, il sera "généralisé " dans la perception esthétique ensuite. Paradoxalement donc, l'univers discontinu du film est celui de la reconnaissance, tandis que c'est la parole poétique qui organise l'univers de la vision (l'univers de l'art) à travers le jeu différentiel qu'elle établit entre l'intériorité et l'extériorité. Le chemin qu'emprunte le cinéma est inverse à 
celui de la poésie. Les deux arts peuvent s'influencer, condamnés toutefois à rester séparés et à travailler chacun son propre matériau $^{29}$.

Peu importe qui des deux protagonistes a raison; nous n'avons pas de solution à apporter. On peut croire qu'Eisenstein a surestimé tandis que Chklovski a sous-estimé les possibilités du cinéma et de son code. Leur débat nous intéresse dans la mesure où il vise à décrire les rapports cinéma-littérature en termes généraux : discontinuité-continuité, différentialité-unité, etc., et permet donc d'enrichir la double démarche habituelle qui consiste à analyser d'un côté, la topique commune (thèmes, motifs, schémas narratifs) et de l'autre, les techniques présumées filmiques (montage, plans, points de vue).

\section{Le cinématisme dans la littérature}

Avant de conclure, il nous faut tester l'idée du cinématisme dans la littérature. Revenons à Zamiatine. À la différence de ses illustres contemporains, il n'opère pas avec un appareil théorique élaboré, il a tendance à schématiser les problèmes soulevés par l'époque. Rappelons également qu'au moment où il formule ses opinions, le débat ne fait que s'amorcer. Il y occupe une position "médiane». En bon disciple du symbolisme et comme Eisenstein plus tard, il bâtit (surtout dans ses cours sur la prose) un système hiérarchisé des arts pour lequel il tente d'imaginer un "code de synthèse ". Ce rôle, il le réserve à la littérature capable de représenter toutes les données sensibles (étant à la fois peinture, musique, architecture) et de les intégrer par l'émotion et par la conceptualisation ${ }^{30}$. Opposé au scepticisme de Chklovski pour ce qui concerne la possibilité même d'une telle "synthèse", il croit comme lui à la supériorité du "code littéraire». Qui plus est, il semble relier la notion moderniste de Vitesse à l'évidence, à la bidimensionnalité qui empêche la profondeur: en cela aussi, il se rapprocherait encore de Chklovski. Voyons plutôt comment la position que nous venons d'esquisser est réfractée dans sa prose.

Prenons un récit. Le Pêcheur d'hommes (Lovec celovekov) développe une intrigue secondaire des Insulaires (Ostrovitjane, 1918), œuvre majeure de Zamiatine qu'il rapporta de l'Angleterre et 
dont la parution le gratifia immédiatement du statut de maître prosateur. L'extrait que voici ouvre Le Pêcheur d'hommes (1989, p. 123):

Le plus beau dans la vie, c'est le délire, et le plus beau des délires, c'est d'être amoureux. Dans le brouillard matinal, trouble comme l'amour, Londres délirait. Rose laiteux, les yeux clos, Londres voguait, peu importe où.

Colonnes légères des temples druidiques, hier encore cheminées d'usine; arcs aériens des viaducs en fonte; ponts d'une île mystérieuse vers une autre île mystérieuse; cous cambrés des énormes cygnes-grues d'un noir antédiluvien qui vont se mettre à fouiller le fond à la recherche d'une proie. Effarouchées, des lettres d'or ronflantes se sont éparpillées au soleil — "Rolls-Royce automobiles" - puis se sont éteintes. De nouveau, les alentours calmes, troubles: dentelles des tours embrumées, toile d'araignée frémissante de fils métalliques, lente ronde des tortues-maisons somnolentes qui passent. Et, tel un axe immobile, le gigantesque phallus de pierre de la colonne Nelson.

Au fond de la mer rose laiteuse l'organiste Bailey voguait [...]. Il s'arrêtait devant les vitrines. Voici des bottes $[\ldots]$.

Ce vaste panorama en plongée frappe d'abord par ses contrastes de lumière: entre le brouillard transpercé par le soleil et l'or électrique des réclames, l'air profond comme une mer rose et le dessin au noir des formes verticales et horizontales qui quadrillent la silhouette de la métropole. La perspective et l'échelle changent, le regard descend du ciel jusque dans la rue, s'approche de plus en plus d'un personnage dont on verra en gros plan les "lèvres grosses, et sans doute molles comme celles d'un poulain». Brutalement focalisé sur lui, le point de vue bascule: des vitrines, des bottes envahissent le champ de vision. L'ensemble de la représentation est animé par un mouvement pluriel donné comme au ralenti: Londres flotte autour de son axe phallique, ses contours se dessinent progressivement, les maisons rampent comme des tortues, le héros avance avec paresse.

Le tournoiement de la ville remplie à ras bord d'un brouillard lumineux rend étranges, mystérieuses les formes quotidiennes 
(le procédé formaliste de la «distanciation » est ainsi thématisé et exposé, "mis à nu"). D’autre part, le récit transforme ce mouvement en une métaphore spatiale de la temporalité: les objets prennent l'apparence de ce qu'ils ne sont pas, ouvrent des couloirs dans le temps, vers l'époque des druides ou l'âge antédiluvien.

Voici un autre passage du récit, une autre vision de la même ville (p. 142) :

Il fait sombre. La porte de la pièce voisine n'est pas bien fermée. À travers la fente de la porte, un rai de lumière se projette sur le plafond: on marche avec une lampe, il est arrivé quelque chose. Le rai bouge de plus en plus vite, et les murs sombres s'éloignent de plus en plus, vers l'infini, et cette chambre, c'est Londres, et il y a des milliers de portes, des lampes s'agitent, des rais de lumière s'agitent sur le plafond. Et tout cela n'est peutêtre qu'un délire [...] Il est arrivé quelque chose. Le ciel noir au-dessus de Londres s'est fendillé en morceaux: des lignes, des triangles, des carrés blancs - délire géométrique silencieux des projecteurs.

Contraste violent avec le début du récit: la ville est plongée dans le noir; elle est toujours en mouvement, le rythme s'accélère. La nuit ressemble à une chambre dont les murs bougent espace fantastique qui se contracte et se gonfle - on pense à la "chambre-cinéma» de Dziga Vertov (1923, p. 140): "[...] je t'ai mis dans une chambre étonnante qui n'a jamais existé auparavant et que je viens aussi de créer. Cette chambre a 12 murs que j'ai empruntés aux différentes parties du monde. " Ensuite, la chambre se démultiplie; le rai de lumière commence ses évolutions inquiétantes. Un souvenir d'enfance, une association imaginée constituent un prisme subjectif qui filtre et déforme l'image: le délire et l'illusion se mélangent au réel graduellement dévoilé et déchiffré. Énigmatique - le mot définit également l'ambiance de l'extrait précédent -, le visible se charge d'un symbolisme dans lequel nous reconnaissons l'antithèse zamiatinenne du chaos et de l'ordre. L'espace nocturne de la géométrie en folie signifie le temps intérieur, le temps en suspension, celui des peurs enfantines et des angoisses sociales. Sa collision avec le 
temps extérieur - c'est la guerre, Londres subit une attaque aérienne - conduit à l'extraordinaire, le quotidien tourne au délire à l'instar de la ville bombardée, le désir explose et se laisse momentanément combler, le confortable rite social reprendra ses droits, mais l'univers de l'ordre aura révélé le chaos qui le sous-tend. C'est ainsi qu'on peut résumer l'intrigue (érotique) du Pêcheur d'hommes. Les descriptions zamiatiniennes, plus qu'un fond ou une mise en ambiance, relaient, par des moyens plastiques et dynamiques, des thèmes et des motifs structurels que le récit déroule sur le plan narratif, sémantique, etc.

Dans les extraits que nous venons de voir, ces moyens se déploient en tant que la confrontation permanente de la lumière et du mouvement. En couleurs ou en noir et blanc, en aplats ou en demi-teintes, la lumière submerge l'espace, elle le modèle entièrement; l'ubiquité de la lumière et son jeu - éclat, reflet, éclaboussure, danse de projecteurs -, justifie que l'on s'interroge sur le rapport de cette prose à l'art qui, en allemand, se nommait justement "jeu de lumière ", Lichtspiel. Le mouvement aux rythmes contrastés est celui de la lumière, mais aussi celui du regard et celui de tout objet capté dans le champ de vision; il est multiple et relatif. Sa simultanéité se trouve matérialisée dans le récit par l'action parallèle et la narration qui suit en alternance deux personnages qui progressent dans la ville à un rythme différent et avec des objectifs opposés.

Simultanéité donc, fragmentation et différenciation, relativité et imbrication du temps et de l'espace, «spatialisation du facteur temporel ": tels sont les principes de représentation que nous venons de trouver dans l'écriture de Zamiatine. Si l'on en croit l'historien de l'art Arnold Hauser, ils caractérisent une conception du temps née après Bergson, stimulée par la révolution scientifique et qui conditionne à son tour l'art moderne: le cinéma permettrait leur réalisation stylistique la plus accomplie ${ }^{31}$.

À noter aussi une transformation presque imperceptible, mais capitale: la nuit qui, grâce à la métaphore de la "chambre", s'étend dans une profondeur mobile, s'aplanit d'un coup: elle se réduit au plafond du ciel noir, une surface où les rais de lumière dessinent "des lignes, des triangles, des carrés blancs" — un tableau quasi suprématiste ou plutôt quasi constructiviste. La 
contradiction qui oppose ainsi la bi- et la tridimensionalité rend encore plus intense la dynamique de la représentation, elle exprime une tension entre le visible et l'imaginaire, le réel et le fantastique. On peut la voir comme l'équivalent de l'opposition fondatrice au cinéma entre la planéité matérielle de l'écran et l'illusion de la profondeur de l'image.

La dernière citation nous permettra de placer un fond sur lequel la nouveauté de cette nouvelle manière devra se détacher. Nous empruntons un fragment à Tolstoï (1972, p. 447) qui reste à nos yeux insurpassable en matière de "synthétisme ":

\begin{abstract}
Debout au milieu de la grange nouvellement construite, Lévine regardait par la porte ouverte, tantôt la poussière sèche et amère du blé qu'on battait, qui s'élevait et retombait sur l'herbe éclairée par le soleil chaud, et sur la paille fraîche qu'on venait de sortir de la grange, tantôt les hirondelles au ventre blanc qui, en sifflant, s'installaient sous le toit, tantôt les paysans qui travaillaient dans la grange sombre et pleine de poussière; et ses idées étaient singulières.
\end{abstract}

Tournoiement de la poussière au goût amer, vol des hirondelles, gestes des travailleurs - plages de lumière et d'ombre qui strient la profondeur de l'image —, sifflements et bruit du blé qu'on bat - chaleur et fraîcheur - toutes les sensations sont condensées en un moment dont la durée se confond avec le mouvement, lui aussi tournant, du regard. Le temps divisé en écoulements simultanés, l'espace rythmé par le jeu du clairobscur, s'imbriquent ici étroitement. Tout annonce déjà la perception moderniste. Mais le récit est continu, il montre la simultanéité graduellement, sans laisser éclater l'objet; la phrase est souple, élaborée en symétrie, la description s'articule au discours auctorial de motivation et d'explication: "Lévine regardait par la porte [...] ses idées étaient singulières" (en russe: strannye, détail intéressant qui souligne le différent statut de la scène dans l'économie du récit, son "extemporalité»). En comparaison, le style de Zamiatine incarne la discontinuité avec son phrasé sur-rythmé, elliptique, avec ses défilés de métaphores qui s'insèrent dans l'énoncé sans préavis en brouillant les niveaux de réalité. 
Lopposition discontinuité-continuité est au fondement de la réflexion sur le temps. L'écriture de Zamiatine est elliptique dans son ensemble; elle procède par l'effacement des explications causales (constituant la construction qui tient tout l'univers de Tolstoï). Les réactions des personnages, leurs actions et les événements qui se produisent autour d'eux n'ont plus de motivation explicite - elle est remplacée par la rhétorique poétique. La continuité du récit est brisée, le temps se fractionne, mais leur unité est rétablie par le lecteur. D'une certaine manière, cette dialectique peut être mise en parallèle avec la relation antithétique, sans laquelle le cinéma n'aurait pas été possible: la discontinuité se présente à la fois comme une qualité matérielle première du film et comme son procédé de base sous la forme d'enchaînement de plans; elle doit être surmontée par la perception du spectateur, par sa perception visuelle en même temps que par son "discours intérieur" (selon le terme d'Eikhenbaum). Le spectateur devient «le foyer de l'énonciation filmique" (Albera, "Introduction ", 1996, p. 19) ${ }^{32}$ de façon analogue au lecteur qui s'affirme comme l'instance énonciative en remplissant les lacunes et redressant les déformations du texte zamiatinien (ou plus généralement, moderniste).

Le détour par l'exemple zamiatinien nous permet de nuancer notre interprétarion des jeux d'influence entre le cinéma et la littérature. Lorsque Le Pêcheur d'hommes est écrit, en 1916-1918, peu de cinéastes peuvent se targuer de savoir utiliser aussi radicalement et avec une telle dextérité les contrastes visuels, le point de vue mobile, les changements rapides de plans. Mais il est également vrai que peu d'écrivains savent montrer l'imbrication fondamentale du temps et de l'espace d'une manière aussi directe et condensée que le fait Zamiatine. C'est l'univers einsteinien qu'il décrit. Ce qui constitue d'ailleurs la raison d'être de sa poétique synthétiste qui, directement ou non, laisse une empreinte décisive sur la littérature de son époque.

Eisenstein avait raison de parler de l'esprit cinématographique propre à la littérature. Il n’a pas voulu le percevoir dans la littérature qui évoluait autour de lui; nous avons démontré cependant qu'une partie au moins de celle-ci correspondait bien à ses critères, bien marquée par des traits "cinématiques": jeu de lumière 
et de vitesse, fragmentation de l'espace-temps, multiplication des dimensions, tension entre le simultané et le séquentiel...

En guise de conclusion, nous proposons de repérer les frontières (fluides) entre les notions: le cinématisme recouvrirait les aspects dynamiques et plastiques de la littérature du passé antécinématographique; le cinématographisme de la littérature contemporaine du cinéma qui y renvoie directement ou imite explicitement ses procédés (le ciné-récit d'un Jules Romains ou d'un Ehrenbourg); enfin, le cinématomorphisme d'une écriture moderniste (Zamiatine, Grine) qui traduit moins l'influence immédiate du cinéma que les nouvelles conceptions du mouvement, du temps, de l'objet, mais qui se retrouve à côté de lui dans ses tentatives d'exploration du monde.

Et terminons sur ce souhait: puisse la recherche «intermédiale " pénétrer plus avant dans le champ ouvert par le dialogue et la compétition entre le cinéma et la littérature dans la Russie des années vingt.

\section{Université de Lausanne}

\section{NOTES}

1. Une partie des observations et des thèses proposées ici ont été présentées dans mon article "Temps-mouvement, espace-lumière. Effet-cinéma, Zamiatine et la prose des années vingt ", Revue des études slaves, LXXI/3, (1999).

2. Voir par exemple S. Han-Magomedov, «Dizajn... v tvorcestve hudoznika Aleksandra Rodcenko", dans A. Rodcenko, Stati. Vospominanija. Avtobiograficeskie zapiski. Pisma, Moscou (Sovetskii khudozhnik, 1982, p. 10-15).

3. Voir O. Molok, V. Kostin, “Ob odnoj idee "buduscego sinteza zivyh iskusstv". Po materialam pisem V.N.Cekrygina k N.N.Puninu nacala 20-h gg. ", Sovetskoe iskusstvoznanie '76 (Moscou, vyp. 2, 1977).

4. Voir la préface et la bibliographie de F. Albera (direction), Les Formalistes russes et le cinéma (Paris: Nathan, 1996).

5. Voir par exemple L. Losev, "Marina Tsvétaiéva", dans E. Etkind, G. Nivat, I. Serman, V. Strada (direction). Histoire de la littérature russe. Le XX siècle. La Révolution et les années vingt (Paris: Fayard, 1988, p. 129-131).

6. Par exemple pour les films de B. Barnet La jeune fille au carton à chapeau er La Maison de la place Troubnaïa.

7. Voir par exemple A. Cernysev, Russkaja dooktjabr'skaja kino-zurnalistika (Moscou, 1987, p. 56).

8. Voir A. Kaes (direcrion), Kino-Debatte. Texte sum Verhältnis von Literatur und Film. 1909-1929, Tübingen, 1978; voir aussi le chapitre sur "Littérature et cinéma", dans J.-M. Glikson, L'Expressionnisme littéraire (Paris: PUF, 1990). 
9. Voir dans Ivan Goll, Le Nouvel Orphée (Paris, 1923).

10. L. Andreev, «Administrativnyj vostorg. Kinematograficeskij rasskaz o bestalannom Vasen'ke", Polnoe sobranie socinenij (t.7, SPb, 1913). Voir fragments traduits en français sur le site: www.unil.ch.

11. "Donogoo-Tonka" dans Kino-Fot, $\mathrm{n}^{\circ} 4$ (1922) p.12.

12. Pour la mise en pages voir surtout, dans la même livraison de $L E F$ où parut l'article de Kouchner (n`3, 1923), D. Vertov, "Kinoki"; P. Neznamov, "Zolotosit'e i galuny".

13. I. Erenburg [Ehrenbourg], Materializacija fantastiki (1927), Fabrika snov (1931).

14. K. Cukovskij [Tchoukovski], "Nat Pinkerton" (1906), dans Sobranie socinenij (vol. 6, Moscou, 1969). Voir extraits en français: www.unil.ch.

15. O. Henry y est transcrit en russe "à l'irlandaise", comme "O'Henry".

16. Voir O. Mandel'stam, "Kinematograf", dans Sobranie socinenij (t.1, New York, 1967); voir en français dans S. Fauchereau, L'Avant-garde russe. Futuristes et acméistes (Paris: Seuil, 1990, p. 109-110).

17. K. Cukovskij, "Nat Pinkerton ", dans Sobranie socinenij, déjà cité.

18. Voir notamment V. Sklovskij [Chklovski], Ejzenstejn (Moscou, 1973).

19. Cet auteur a écrit une série de textes "documentaires", comme Le Peuple en guerre, qui mélangent dialogues, conversations, phrases, dictons donnés comme authentiques, recueillis par l'auteur, sans un mot de commentaire.

20. F. Albera, "Introduction", dans Eisenstein (1980, p. 9).

21. Sur l'application de ce terme aux arts plastiques, voir F. Albera dans Eisenstein, 1980 , p. 8-9; sur son élargissement, voir, par exemple, N. Klejman [Kleiman], préface à Eisenstein, 1999b, p. 106.

22. Voir Eisenstein, 1999b, p. 109-128.

23. L. Kulesov, Osnovy kinorezissury (Moscou: Kinoizdat, 1941, p. 66-67).

24. M. Romm, "O kino i horosej literature" (1954), dans M. Romm, Besedy o kino, Moscou, 1964. Une partie de ce texte a été traduite en français sous le titre "Littérature et cinéma" dans "Recherches soviétiques" dans Cinéma, n³, 1956, p. 103-126.

25. Voir Eisenstein, 1989b.

26. V. Nabokov, Gogol (Paris: Union générale d'Éditions, 1995, coll. «10/18»).

27. Voir "Cetvertoe izmerenie v kino" [1929], dans Eisenstein, 1964-1971, t. II, p. 59.

28. L. Sabaneev, Skrjabin (Moscou, 1916; Muzyka reci, Moscou, 1923).

29. Voir Chklovski, 1985, p. 113-115.

30. Voir Zamiatine, 1990, p. 198-267.

31. A. Hauser, Histoire sociale de l'art et de la littérature (Paris: Le Sycomore, 1982, t. IV, p. 215).

32. Voir aussi B. Eikhenbaum, "Problèmes de ciné-stylistique", dans Albera, 1996, p. $44-45$.

\section{OUVRAGES CITÉS}

N. B. : les ouvrages cités d'Eisenstein se retrouvent, en fin de numéro, dans une liste particulière.

Albera, François (direction). Les Formalistes russes et le cinéma. Paris: Nathan, 1996.

Belyj [Biély], Andrej. "Sinematograf" dans Arabeski, Moscou, 1911.

Boulgakov, Mikhail. Diablerie, suivi de Les CEufs fatidiques et autres récits. Lausanne: L'Âge d'Homme, 1971. 
Cendrars, Blaise. La Fin du monde filmée par l'ange Notre-Dame. Paris: La Sirène, 1919.

Erenburg [Ehrenbourg], Ilya. A vse-taki ona vertitsja. Berlin, 1922.

Gogol, Nikolaï. Tarass Boulba. Moscou : Éditions en langues étrangères, s.d.

Grine, Alexandre. L'Attrapeur de rats. Lausanne: L'Âge d'Homme, 1972.

Heil, Jerry. "Russian Writers and the Cinema" dans Russian Literature, Amsterdam, vol. XIX-II (1986).

Kusner [Kouchner], Boris. "Izopovest' " dans $L E F, \mathrm{n}^{\circ} 3$ (1923).

Lidin [Lidine], Vladimir. Povesti o mnogih dnjah. Berlin : Ogon'ki, 1923.

Rodcenko [Rodtchenko], Aleksandra. "Sarlo" dans Kino-fot, $\mathrm{n}^{\circ} 3$ (1922).

Sklovskij [Chklovski], Viktor. Za sorok let. Moscou: Iskusstvo, 1965.

Tolstoï, Léon. Anna Karenine. Paris: Le Livre de poche, 1972, t. II.

Vertov, Dziga. “Kinoki. Perevorot » dans $L E F, \mathrm{n}^{\circ} 3$ (1923).

Zamiatine, Evguéni. Le Pếcheur d'hommes. Paris-Marseille: Rivages, 1989. 